\title{
CERTAIN IDEMPOTENTS LYING IN THE CENTRALIZER OF THE GROUP OF UNITS
}

\author{
R. P. HUNTER ${ }^{1}$
}

\begin{abstract}
Let $S$ be a compact connected monoid of dimension $\boldsymbol{n}$ having $\boldsymbol{G}$ as a connected group of units. Let $B$ be a closed subgroup outside of the minimal ideal. The maximum dimension possible for the product $B G$ is $n-1$. If this maximum is attained by $B G$ and $G B$ and both are Lie groups then $B$ meets the centralizer of $G$.
\end{abstract}

Let $S$ be a compact connected monoid whose group of units $G$ is connected. Our purpose here is to show that certain subgroups of $S$ must meet, and in some cases lie in $Z(G, S)$, the centralizer of the group of units. These results stem in part from the manner in which an (algebraically) irreducible submonoid is embedded. For example, it is shown [1] that an irreducible submonoid cannot have dimension at the unit exceeding $\operatorname{dim} S-\operatorname{dim} G-$ 1. There, the important item was the structure of the product $G B$ where $B$ was a subgroup of $S$.

If the closed subgroup $B$ lies outside of the minimal ideal the maximum dimension possible for $G B$ is $\operatorname{dim} S-1$. It is this case which we shall pursue. If $S$ is finite dimensional with $G$ and $B$ both Lie group then the condition

$$
\operatorname{dim} B G=\operatorname{dim} G B=\operatorname{dim} S-1
$$

will cause $B$ to meet $Z(G, S)$. If $G$ happens to be semisimple, $\operatorname{dim} S-2$ will suffice in place of $\operatorname{dim} S-1$.

Here, we shall be concerned with the products of the form $G B G$. We show that if $B$ is normal in its maximal subgroup then $G B G$ is the space of a fiber bundle where the fiber is the homogeneous $B G$ and the base is a quotient of $G$.

Suppose now that $S$ and $G$ are as above and that $e$ is an idempotent belonging to the compact subgroup $B$. Further, suppose that $B$ is disjoint from the minimal ideal as well as $G$.

In order to show that a given $e$ is in $Z(G, S)$, we must show that $G e \subseteq e S$ and $e G \subseteq S e$. This is equivelent to $G e \cup e G \subseteq H_{e}$-the maximal subgroup of $e$. Another equivalent formulation is that $G e \subseteq B G$ and $e G \subseteq G B$. We first concentrate upon the condition that $G e \subseteq B G$. The following definition is appropriate:

Definition. Let $Y=Y(B, G)=\{g \mid g \in G, g B G=B G\}$. Clearly, $Y$ is a

Received by the editors July 17, 1975 and, in revised form, January 26, 1978.

AMS (MOS) subject classifications (1970). Primary 22A15; Secondary 22C05, 22 E20.

${ }^{1}$ With the support of the National Science Foundation MPS 74-07279. 
closed subgroup of $G$. The elements of $Y$ may be described in a number of ways: (1) $g \in Y$; (2) $g B G \subseteq B G$; (3) $g B \subseteq B G$; (4) $g e \in \cap\{B G b: b \in B\}$. Consider, however, the following condition

$$
g B G \cap B G \neq \varnothing .
$$

There is no apparent reason to suppose that this condition will place $g$ in $Y$. A moment's reflection, however, indicates that this condition is what is needed so that the sets $\{g B G\}, g \in G$, do form a decomposition.

Definition. We shall say that $B$ is meshed with $G$, (on the left), if $g B G \cap B G \neq \varnothing$ implies $g \in Y$, i.e. $g B G=B G$.

The appropriateness of this notion is shown in the following proposition which is fundamental.

Proposition 1. Let $G$ be the group of units of a compact monoid and let $B$ be a compact subgroup outside of $G$ and the minimal ideal. If $B$ is meshed with $G$ then there is an open surjection $p: G B G \rightarrow G / Y$ given by $p(g B G)=g Y$ with $p^{-1}(g Y)=g B G$. Thus, $(G B G, p, G / Y)$ is a fiber space. If $\operatorname{dim} G / Y$ is finite then $q: G \rightarrow G / Y$ admits a local cross-section and the fiber space $p: G B G \rightarrow$ $G / Y$ is a locally trivial fiber bundle with fiber $B G$. The associated bundle is $G \rightarrow G / Y$.

Proof. Since $B$ is meshed with $G$ it is clear that $p$ is well defined and that $p^{-1}(g Y)=g B G$. The fibers $[g G B]$ are clearly all homeomorphic with $B G$. Let 0 be an open set in $G B G$ and let $V$ and $W$ be such that $V \times W$ is the inverse image of 0 under the multiplication $G \times B G \rightarrow G B G$. Now $p(V B G)$ $=V Y$ which is certainly open in $G / Y$ since $G \rightarrow G / Y$ is open. But $p(0)=$ $p(V B G)$. Thus $p$ is open. Suppose now that $G / Y$ is of finite dimension. We know from [5] that local cross-sections exist. Thus let $U$ be an open set in $G / Y$ and $s: U \rightarrow G$ a continuous map with $q s(u)=u$ for $u \in U$. Define the homeomorphisms $h$ and $f$ by

$$
\begin{array}{ll}
h: U \times Y \rightarrow q^{-1}(U), & h(u, y)=s(u) \cdot y, \\
f: U \times B G \rightarrow p^{-1}(U), & f(u, x)=s(u) \cdot x .
\end{array}
$$

Then the following diagrams commute. The first indicates the bundle structure of $q: G \rightarrow G / Y$, which is well known and the second that of $p$ : $G B G \rightarrow G / Y$.
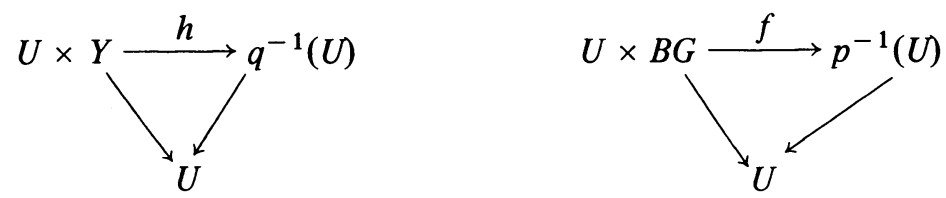

The unmarked arrows being projections and $p$ and $q$ appropriately cut down. Now let $w \in U \cap U_{0}$ with $U_{0}$ and $s_{0}$ playing the role of $U$ and $s$. Combining the transition functions

$$
f_{0}^{-1} f(w, x)=\left(w, s_{0}^{-1}(w) s(w) x\right) .
$$


Hence the associated transformations of the fiber are of the form $x \rightarrow$ $x_{0}^{-1}(w) s(w) x$ with $s_{0}^{-1}(w) s(w) \in Y$. These are the transformations of the principal bundle $g: G \rightarrow G / Y$. By definition, the associated bundle has $G / Y$ as base and $Y$ as fiber. We see that $q: G \rightarrow G / Y$ is indeed the associated bundle. The condition that $B$ be meshed with $G$ is not unreasonable. We see this now.

Lemma 1. If eGe $\cap H_{e}$ lies in the normalizer of $B$ in $H_{e}$ then $B$ is meshed with $G$. In particular, if $C$ is the component of $e$ in $H_{e}$ then $C$ and $G$ are meshed.

Proof. Suppose $g B G \cap B G \neq \varnothing$. Let $x_{1}, x_{2} \in B, y_{1}, y_{2} \in G$ with $g x_{1} y_{1}=$ $x_{2} y_{2}$. Clearly $g x_{1} G=x_{2} G$ and $B g x_{1} G=B G$. We may then write

$$
B g x_{1} G=B e g e x_{1} G=e g e B x_{1} G=e g B G=B G .
$$

But $g B G$ lies entirely in the $R$-class of $e$ since $g B G$ meets $B G$. But then $e g B G=g B G$ so that $g B G=B G$.

When studying the fiber space $G B G \rightarrow G / Y$ we are often concerned with the conclusion that $G / Y$ be nondegenerate. In the important case in which $B$ is a group component we have the following:

LEMMA 2. Let $S$ be a compact monoid whose group of units $G$ is connected. Let $C$ be the component of $e$ in $H_{e}$, where $e^{2}=e$. If $G C \neq C$ then $G / Y \neq \varnothing$.

PRoof. First, $G C$ lies in the $\mathcal{L}$-class of $e$. We cannot have $G C \subseteq H_{e}$ since this would imply $G C=C$. Thus, part of $G C$ lies outside of the $\Re$-class of $e$. But the last contains $C G$. Thus $G C \neq C G$ so that $G C G \neq C G$. Then, by definition, $G / Y \neq 0$ where $Y=\{g \mid g C G \subseteq C G\}$.

Let us note the following fact: If $G$ is a group of units and $B$ is a subgroup then $B G=B$ implies $G B$ is a left simple semigroup and hence a left group. In effect, $G B g b=G B b=G B$.

In order to continue our study of $G B G$ we recall some material from [1].

Definition. Let $W=\{g \mid g B=B, g \in B\}$.

Observe that $g \in W$ if and only if $g B \subseteq B$ if and only if $g e \in B$.

LEMMA 3. The group $G \times B$ acts on Se via $(b, g) \cdot x=g x b^{-1}$. Moreover, the map $g \rightarrow(g, g e)$ takes $W$ isomorphically onto the isotropy group $(G \times B)_{e}$ at $e$.

Proof. Observe that $w \rightarrow$ we is a homomorphism. Furthermore, $(g, b) \in$ $(G \times B)_{e}$ if and only if $b=g e$.

LEMMA 4. Multiplication, $(g, b) \rightarrow g \cdot b$ is a principal fibration $G \times B \rightarrow G B$, with fiber $W$. This fibration is the quotient map of a homogeneous space.

Proof. Since $G B$ is the orbit of $G \times B$ at $e$ the map is equivalent to the quotient map defined by $(G \times B)_{e}$.

For our purposes it is necessary to determine $\operatorname{dim} G B G$. This is now given:

Proposition 2. Let $B$ and $G$, given as above, be meshed. If $G$ and $B$ are finite dimensional then $\operatorname{dim} G B G=\operatorname{dim} B G+\operatorname{dim} G / Y$.

Proof. From the preceding, $B G$ is locally the product of a zero 
dimensional compact set and a cell of dimension equal to $\operatorname{dim} B G$. Likewise $G / Y$ has its dimensions determined by a cell of the same dimension as $G / Y$. Since $G B G$ is locally a product of open sets from $B G$ and $G / Y$, we have the desired conclusion.

Let us note that if $G$ is connected and $\operatorname{dim} G / Y=0$, which is the same thing as $\operatorname{dim} B G=\operatorname{dim} G B G$, then $G e \subseteq B G$, by the definition of $Y$. Thus, the larger the dimension of $B G$ the more likely our desired conclusion.

Now let $S$ be a compact connected finite dimensional monoid having a group of units $G$ and a compact subgroup $B$ outside of the minimal ideal. Then the maximum dimension possible for $B G$ is $\operatorname{dim} S-1$. One can see this in two different ways. Using the action of $B \times G$ upon $B S$ one can cite [2] directly. On the other hand, $B G$ is a homogeneous space of $B \times G$ and so has nonzero element in $H^{k}(B G)$ where $k=\operatorname{dim} B G$. This is ruled out by [7].

Definition. With $B$ and $G$ as above, not necessarily meshed however, we shall say that $B G$ is of maximal dimension if $\operatorname{dim} B G=n-1$.

LEMMA 5. If $B G$ is of maximal dimension then $B$ is meshed with $G$.

Proof. If $C$ denotes the identity component of $e$ in the group $H_{e}$ then clearly $\operatorname{dim} C G=\operatorname{dim} B G$. However, from the remarks before, $C \times G$ yields the homogeneous space $C G$ and $B \times G$ yields the homogeneous space $B G$. But a compact connected homogeneous space cannot contain properly another compact homogeneous space of the same finite dimension.

Suppose now that $S, G, B$ are as above with $\operatorname{dim} S=n$ and $B G$ of maximal dimension. Then we either have $\operatorname{dim} G B G=n-1=\operatorname{dim} B G$ or we have the (unlikely) situation $\operatorname{dim} G B G=n$. In view of a number of considerations to follow, an appropriate way of eliminating the second possibility is that $G B G$ have nonzero cohomology in its top dimension, i.e. $H^{\operatorname{dim} G B G}(G B G) \neq 0$. This is due to a well-known result of Wallace. See [7] or [4].

Thus, we may state the following.

LEMMA 6. Let $S$ be a compact connected monoid of finite dimension having a connected group of units $G$. Let $B$ be a compact connected subgroup (outside of the minimal ideal) such that $G B$ and $B G$ are of dimension $\operatorname{dim} S-1$. Then if $H^{k}(G B G) \neq 0$, where $k=\operatorname{dim} G B G$, the subgroup $B$ meets $Z(G, S)$.

Observe now that if $B$ is meshed with $G$ and both are Lie groups then $G B G$ is a manifold. In effect $G B G$ is fibered over the manifold $G / Y$ by the manifold $B G$. Thus, in this case, $H^{\operatorname{dim}(G B G)}(G B G) \neq 0$.

COROLlaRY 1. Let $S$ be a compact connected monoid of finite dimension whose group of units $G$, is a connected Lie group. Suppose $e$ is an idempotent outside of the minimal ideal and that $C$ the group component of $e$ is also a Lie group. If $\operatorname{dim} G B=\operatorname{dim} B G=\operatorname{dim} S-1$ then e belongs to $Z(G, S)$.

If $G$ is semisimple then

$$
\operatorname{dim} B G \geqslant \operatorname{dim} S-2 \leqslant \operatorname{dim} G B
$$

imply that e belongs to $Z(G, B)$. 
Proof. For the second claim, recall that a compact connected semisimple Lie group cannot yield a homogeneous space of dimension one. Thus, $\operatorname{dim} G / Y$ is at least two unless $G C=C$. We would then have the manifold $G C G$ of dimension

$$
\operatorname{dim} G / Y+\operatorname{dim} C G \geqslant 2+(\operatorname{dim} S-2)=\operatorname{dim} S,
$$

which is impossible.

COROLLARY 2. Let $S$ be a compact connected monoid of dimension $n$, having a connected group of units $G$. If $e$ is an idempotent outside of $G$ and the minimal ideal such that $\operatorname{dim} H_{e}=n-1$ then $e \in Z(G, S)$.

Proof. We note that $G C$ is a homogeneous space of $G \times C$. Were we to have $C$ properly contained in $G C$ we must have $\operatorname{dim} G C=n$, since a homogeneous space which is compact and connected may not contain another of the same finite dimension. Thus $G C=C$ and, in the same way, $C G=C$.

COROLLARY 3. Let $S$ be a compact connected finite dimensional monoid whose group of units $G$ is a connected semisimple Lie group. Let e be an idempotent belonging to neither $Z(G, S)$ nor the minimal ideal. If $C$ the maximal compact connected subgroup at $e$ is a Lie group then $\operatorname{dim} C \leqslant \operatorname{dim} S-3$.

Proof. Since $e \notin Z(G, S)$ we cannot have $G C=C G=C$. Say $G C \neq C$. Then consider the fibering $G C G \rightarrow G / Y$. Since $G C \subsetneq C$ we know that $G C \subsetneq_{+} C G$. Thus the quotient $G / Y$ is nondegenerate. Since $G$ is semisimple $\operatorname{dim} G / Y \geqslant 2$. Now, $\operatorname{dim} G C G=\operatorname{dim} C G+\operatorname{dim} G / Y$. Thus, if $\operatorname{dim} C=$ $\operatorname{dim} S-2$ we would have $\operatorname{dim} G C G=\operatorname{dim} S$ which, as we know, is impossible. Hence $\operatorname{dim} C \leqslant \operatorname{dim} S-3$.

COROLLARY 4. Let $S$ be a compact connected monoid having a group of units $G$ which is a connected Lie group. Let $e$ be an idempotent belonging to neither $Z(G, S)$ nor the minimal ideal. Let $C$ denote the component of $e$ in $H_{e}$ and suppose that $C$ is a Lie group. If either

(1) $\operatorname{dim} C=\operatorname{dim} S-2$ or

(2) $G$ is semisimple and $\operatorname{dim} C=\operatorname{dim} S-3$,

holds then either GC or CG (not both) coincides with C. Thus, say, GC is a left group with $C$ as maximal subgroup.

Proof. Consider again $G C G \rightarrow G / Y$. Suppose first, $\operatorname{dim} C=\operatorname{dim} S-2$. Then if both $G C \neq C$ and $C G \neq C$ we would have $\operatorname{dim} G C G=\operatorname{dim} C G+$ $\operatorname{dim} G / Y$. Since $C \subsetneq C G$ we have $\operatorname{dim} C G \geqslant \operatorname{dim} S-1$. Since $G C \neq C$ we have $G C G \subsetneq C G$ so that $\operatorname{dim} G / Y \geqslant 1$. Thus, $\operatorname{dim} G C G=\operatorname{dim} S$ which is impossible. Now suppose that $\operatorname{dim} C=\operatorname{dim} S-3$ with $G$ semisimple. In this case if $G C \neq C$ and $C G \neq C$ we have

$$
\operatorname{dim} G C G=\operatorname{dim} C G+\operatorname{dim} G / Y \geqslant \operatorname{dim} S-2+2=\operatorname{dim} S .
$$

Again, a contradiction.

ExAmPLE. Let $G$ be the three sphere as a topological group using 
quaternions of norm one. Following [3] let $|G|$ be the space $G$ with left zero multiplication. Then $L=|G| \times G$ is a left group. Let $G$ act on $L$ by $g\left(g^{\prime}, g^{\prime \prime}\right)=\left(g g^{\prime}, g g^{\prime \prime}\right)$ and $\left(g^{\prime}, g^{\prime \prime}\right) g=\left(g^{\prime}, g^{\prime \prime} g\right)$. Then $L \cup G$ is a compact monoid in which $e=(1,1)$, where 1 is the unit of $G$, is not in the centralizer of $G$. Instead of $G$ one may use some homogeneous space of $G$ say $G / K$. Again $G \cup\{|G / K| G\}$ is a monoid. We may take $S=G \cup\{|G / K| G\}$ of dimension five with $G / K$ say $S^{2}$. Now take the cone over $S$ and get a compact connected monoid of dimension six with $e$ having its component of dimension three $=n-3$. Here $e$ is not in the centralizer of the group of units. However the set corresponding to $G C$ is again a left group. See [3] for further details.

As the reader has noted, the fibering of $G B G$ by $B G$ over $G / Y$ shows that the total space has nontrivial cohomology in top dimension if $B$ and $G$ are manifolds, i.e. Lie groups. It thus seems reasonable to conjecture that if $(E, B, F)$ is a fibre bundle with space $E$, base $B$ and fibre $F$ then $B$ and $F$ are compact connected finite dimensional topological groups one could conclude that $H^{n}(E, Z) \neq 0$ where $n=\operatorname{dim} B+\operatorname{dim} F$. (It is known that $H^{\operatorname{dim} B}(B)$ and $H^{\operatorname{dim} F}(F)$ are nonzero over $Z$.) Oddly enough such is not the case.

EXAMPLE. There exists a space $E$ which is a fibre bundle over the circle fibered by the dyadic solenoid with $H^{2}(E, Z)=0$.

Let $K$ be the Klein bottle obtained as usual by identification of the ends of a cylinder $I \times T$ through the diameter. On $T$ the circle this is the map $Z \rightarrow Z^{-1}$. Since this identification map is compatible with the squaring map $Z \rightarrow Z^{2}$ on $T$ we may map $K$ onto itself preserving the base and wrapping each fibre twice. This map $\alpha$ induces $\alpha^{*}$ on $H^{2}(K, Z)$ which is trivial.

Recall that $H^{2}(K, Z)=Z_{2}$. From direct considerations, the kernel of $\alpha^{*}$ is again $Z_{2}$, i.e. all of $H^{2}(K, Z)$. The inverse limit of a square of such spaces $K$ and maps $\alpha$ will yield a fibre bundle $E$ with base a circle and fibre the dyadic solenoid. However,

$$
H^{2}(E, Z)=0 \text {. }
$$

We mention that Floyd has constructed a free action of $p$-adic group on an acyclic space [6].

\section{REFERENCES}

1. L. W. Anderson and R. P. Hunter, A remark on finite dimensional compact connected monoids, Proc. Amer. Math. Soc. 42 (1974), 602-606.

2. J. Day and K. H. Hofmann, Clan acts and codimension, Semigroup Forum 4 (1972), 206-214.

3. K. H. Hofmann and M. Mislove, The centralizing theorem for left normal groups of units in compact monoids, Semigroup Forum 3 (1971), 31-42.

4. K. H. Hofmann and P. S. Mostert, Elements of compact semigroups, Merrill, Columbus, Ohio, 1966. MR 35 \#285.

5. P. S. Mostert, Local cross sections in locally compact groups, Proc. Amer. Math. Soc. 4 (1953), $645-649$.

6. R. F. Williams, The construction of certain 0-dimensional transformation groups, Trans. Amer. Math. Soc. 129 (1967), 140-156.

7. A. D. Wallace, Cohomology, dimension and mobs, Summa Brasil. Math. 3 (1953), 43-54.

Department of Mathematics, Pennsylvania State University, University Park, PennSYLVANIA 16802 